Supporting Information

\title{
Automated Nanoflow Two-Dimensional Reversed-Phase Liquid Chromatography System Enables In-Depth Proteome and Phosphoproteome Profiling of Nanoscale Samples
}

Maowei Dou, ${ }^{1 \dagger}$ Chia-Feng Tsai, ${ }^{2 \dagger}$ Paul D. Piehowski, ${ }^{2}$ Yang Wang, ${ }^{2}$ Thomas L. Fillmore, ${ }^{1}$ Rui Zhao, ${ }^{1}$ Ronald J. Moore, ${ }^{2}$ Pengfei Zhang, ${ }^{2}$ Wei-Jun Qian, ${ }^{2}$ Richard D. Smith, ${ }^{2}$ Tao Liu, ${ }^{2}$ Ryan T. Kelly, ${ }^{1,3 *}$ Tujin Shi, ${ }^{2 *}$ Ying $\mathrm{Zhu}^{1 *}$

${ }^{1}$ Environmental Molecular Sciences Laboratory, Pacific Northwest National Laboratory, Richland, Washington 99354, United States

${ }^{2}$ Biological Sciences Division, Pacific Northwest National Laboratory, Richland, Washington 99354, United States

${ }^{3}$ Department of Chemistry and Biochemistry, Brigham Young University, Provo, Utah 84604, United States

Keywords: Mass spectrometry, multidimensional separations, nanoflow fractionation, proteomics, phosphoproteomics

$\dagger$ Authors contributed equally to this work.

*Corresponding authors:

Dr. Ying Zhu, ying.zhu@pnnl.gov

Dr. Tujin Shi, tujin.shi@pnnl.gov

Dr. Ryan T. Kelly, ryan.kelly@byu.edu 


\section{Table of Contents}

- Fig. S1 Optimization of nanoflow offline fractionation conditions

- Table S1 Automated concatenation process of the nanoflow 2D RPLC platform

- Fig. S2 Identification of unique peptides and proteins with concatenation into 12 fractions

- Fig. S3 The overlap of protein identifications among $100 \mathrm{ng}, 500 \mathrm{ng}$ and $1 \mu \mathrm{g}$ of HeLa tryptic digest samples using the nanoflow 2D RPLC platform

- Fig. S4 The number of identified phosphopeptides eluted into the sample vial without and with $0.01 \% \mathrm{DDM}$ as additive in collection buffer. 

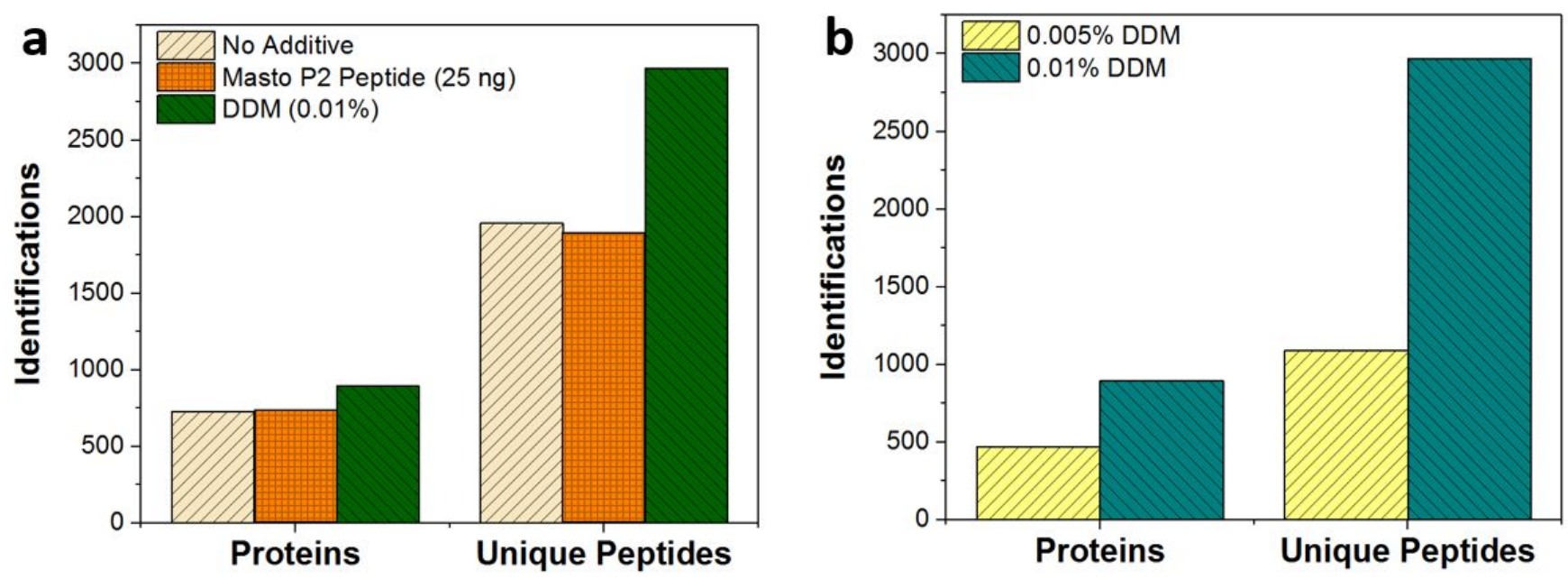

Fig. S1 Optimization of nanoflow offline fractionation conditions using 10-ng protein digest from Shewanella oneidensis MR-1. (a) Peptide and protein identifications with and without additives. (b) Peptide and protein identifications at different concentrations of DDM (collection devices: 96-well plate; collection buffer: Buffer A). 
Table S1 Automated concatenation process of the nanoflow $2 D$

RPLC platform

\begin{tabular}{|c|c|c|}
\hline \multirow{2}{*}{ LC separation (min) } & \multicolumn{2}{|c|}{ Automatic concatenation } \\
\hline & Proteome & Phosphoproteome \\
\hline 1 & F1 & F1 \\
\hline 2 & F2 & F2 \\
\hline 3 & F3 & F3 \\
\hline 4 & F4 & $\mathrm{F} 4$ \\
\hline 5 & F5 & F5 \\
\hline 6 & F6 & F6 \\
\hline 7 & F7 & F1 \\
\hline 8 & F8 & $\mathrm{F} 2$ \\
\hline 9 & F9 & $\mathrm{F} 3$ \\
\hline 10 & F10 & F4 \\
\hline 11 & F11 & F5 \\
\hline 12 & F12 & F6 \\
\hline 13 & F1 & F1 \\
\hline 14 & F2 & F2 \\
\hline 15 & F3 & F3 \\
\hline 16 & F4 & $\mathrm{F} 4$ \\
\hline 17 & F5 & F5 \\
\hline 18 & F6 & F6 \\
\hline 19 & F7 & F1 \\
\hline 20 & F8 & $\mathrm{F} 2$ \\
\hline 21 & F9 & F3 \\
\hline 22 & F10 & $\mathrm{F} 4$ \\
\hline 23 & F11 & F5 \\
\hline 24 & F12 & F6 \\
\hline 25 & $\mathrm{~F} 1$ & F1 \\
\hline 26 & F2 & F2 \\
\hline 27 & F3 & F3 \\
\hline 28 & $\mathrm{~F} 4$ & $\mathrm{~F} 4$ \\
\hline 29 & F5 & F5 \\
\hline 30 & F6 & F6 \\
\hline 31 & F7 & F1 \\
\hline 32 & F8 & F2 \\
\hline
\end{tabular}




\begin{tabular}{|c|c|c|}
\hline 33 & F9 & F3 \\
\hline 34 & F10 & F4 \\
\hline 35 & F11 & F5 \\
\hline 36 & F12 & F6 \\
\hline 37 & $\mathrm{~F} 1$ & $\mathrm{~F} 1$ \\
\hline 38 & $\mathrm{~F} 2$ & F2 \\
\hline 39 & F3 & F3 \\
\hline 40 & F4 & F4 \\
\hline 41 & F5 & F5 \\
\hline 42 & F6 & F6 \\
\hline 43 & F7 & F1 \\
\hline 44 & F8 & F2 \\
\hline 45 & F9 & F3 \\
\hline 46 & F10 & F4 \\
\hline 47 & F11 & F5 \\
\hline 48 & F12 & F6 \\
\hline 49 & $\mathrm{~F} 1$ & $\mathrm{~F} 1$ \\
\hline 50 & F2 & F2 \\
\hline 51 & F3 & F3 \\
\hline 52 & $\mathrm{~F} 4$ & F4 \\
\hline 53 & F5 & F5 \\
\hline 54 & F6 & F6 \\
\hline 55 & F7 & $\mathrm{F} 1$ \\
\hline 56 & F8 & F2 \\
\hline 57 & F9 & F3 \\
\hline 58 & F10 & F4 \\
\hline 59 & F11 & F5 \\
\hline 60 & F12 & F6 \\
\hline 61 & $\mathrm{~F} 1$ & $\mathrm{~F} 1$ \\
\hline 62 & F2 & F2 \\
\hline 63 & F3 & F3 \\
\hline 64 & F4 & F4 \\
\hline 65 & F5 & F5 \\
\hline 66 & F6 & F6 \\
\hline 67 & F7 & F1 \\
\hline 68 & F8 & F2 \\
\hline 69 & F9 & F3 \\
\hline 70 & F10 & F4 \\
\hline
\end{tabular}




\begin{tabular}{|c|c|c|}
\hline 71 & F11 & F5 \\
\hline 72 & F12 & F6 \\
\hline 73 & $\mathrm{~F} 1$ & $\mathrm{~F} 1$ \\
\hline 74 & F2 & $\mathrm{F} 2$ \\
\hline 75 & F3 & F3 \\
\hline 76 & F4 & F4 \\
\hline 77 & F5 & F5 \\
\hline 78 & F6 & F6 \\
\hline 79 & F7 & $\mathrm{F} 1$ \\
\hline 80 & F8 & $\mathrm{F} 2$ \\
\hline 81 & F9 & F3 \\
\hline 82 & F10 & $\mathrm{F} 4$ \\
\hline 83 & F11 & F5 \\
\hline 84 & $\mathrm{~F} 12$ & F6 \\
\hline 85 & $\mathrm{~F} 1$ & F1 \\
\hline 86 & F2 & $\mathrm{F} 2$ \\
\hline 87 & F3 & F3 \\
\hline 88 & F4 & F4 \\
\hline 89 & F5 & F5 \\
\hline 90 & F6 & F6 \\
\hline 91 & F7 & $\mathrm{F} 1$ \\
\hline 92 & F8 & $\mathrm{F} 2$ \\
\hline 93 & F9 & F3 \\
\hline 94 & F10 & F4 \\
\hline 95 & F11 & F5 \\
\hline 96 & $\mathrm{~F} 12$ & F6 \\
\hline
\end{tabular}



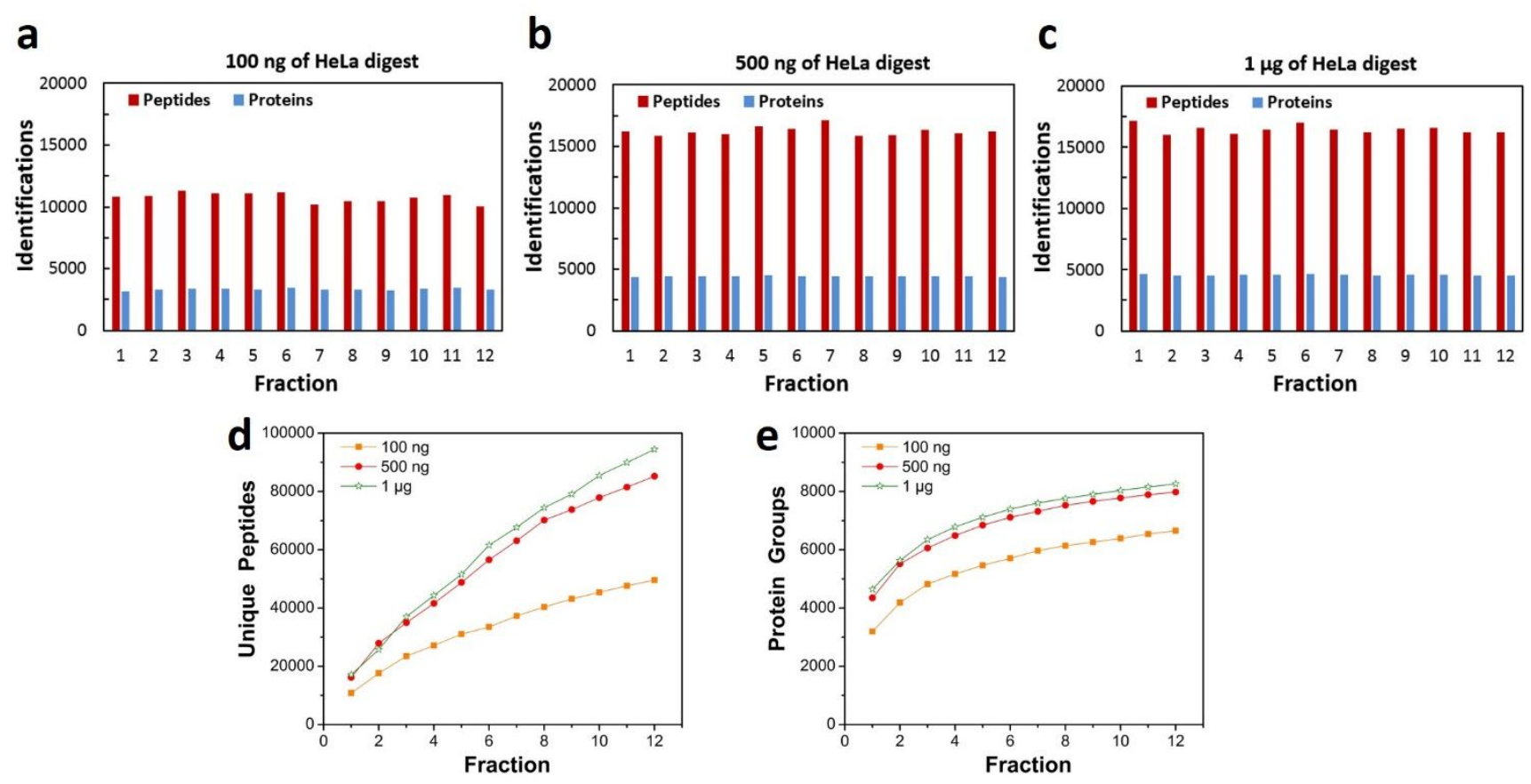

Fig. S2 Identification of unique peptides and proteins with concatenation into 12 fractions. (a-c) Identified unique peptides and proteins per fraction from (a) $100 \mathrm{ng}$, (b) $500 \mathrm{ng}$, and (c) $1 \mu \mathrm{g}$ of HeLa tryptic digest. The identified proteins were nearly uniformly distributed across the fractions, ranging from 3,194 to 3,461 proteins with an average value of 3,321 for $100 \mathrm{ng}$ of HeLa digest, from 4,342 to 4,513 with an average value of 4,424 for $500 \mathrm{ng}$ of HeLa digest, and from 4,501 to 4,673 with an average value of 4,572 for 1 $\mu \mathrm{g}$ of HeLa digest. (d-e) Cumulative numbers of identified unique peptides (d) and protein groups (e) from $100 \mathrm{ng}, 500 \mathrm{ng}$, and $1 \mu \mathrm{g}$ of HeLa tryptic digest corresponding to fraction numbers. 


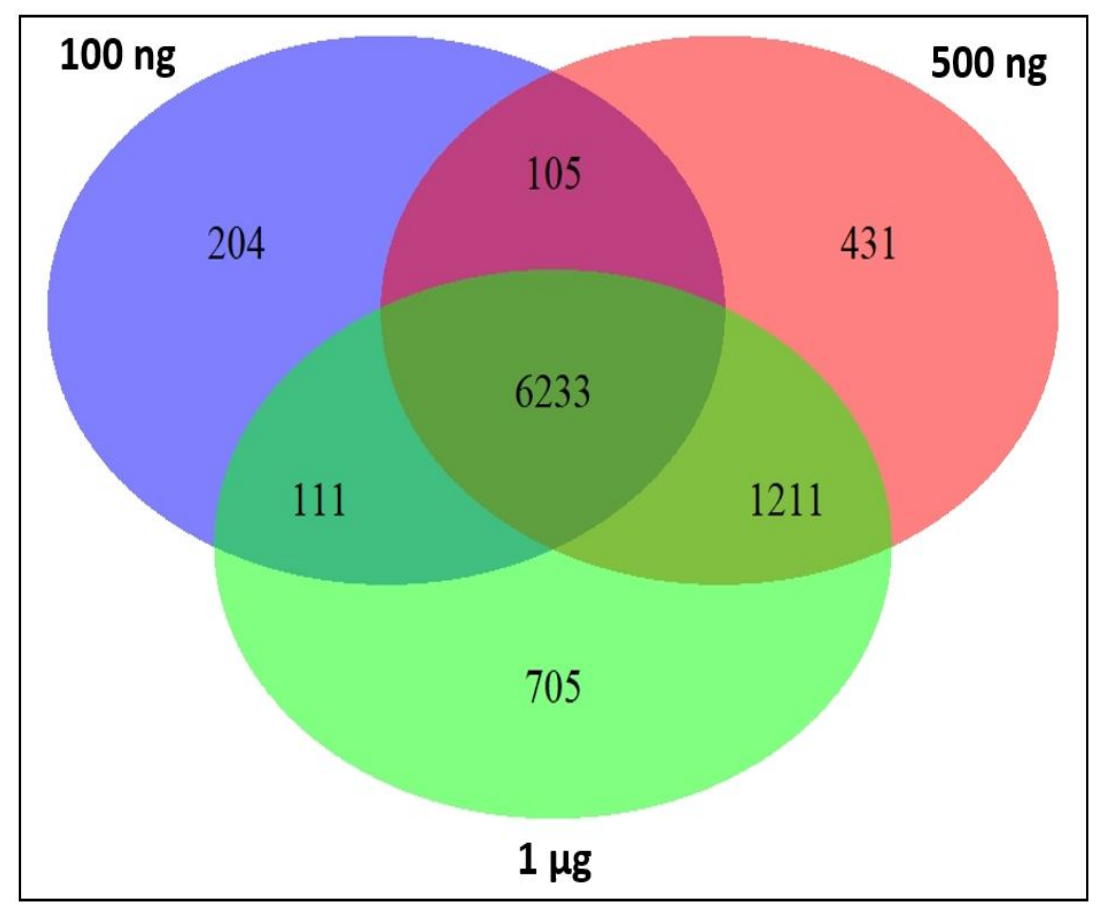

Fig. S3 The overlap of protein identifications among $100 \mathrm{ng}, 500 \mathrm{ng}$ and $1 \mu \mathrm{g}$ of HeLa tryptic digest samples using the nanoflow 2D RPLC platform. 


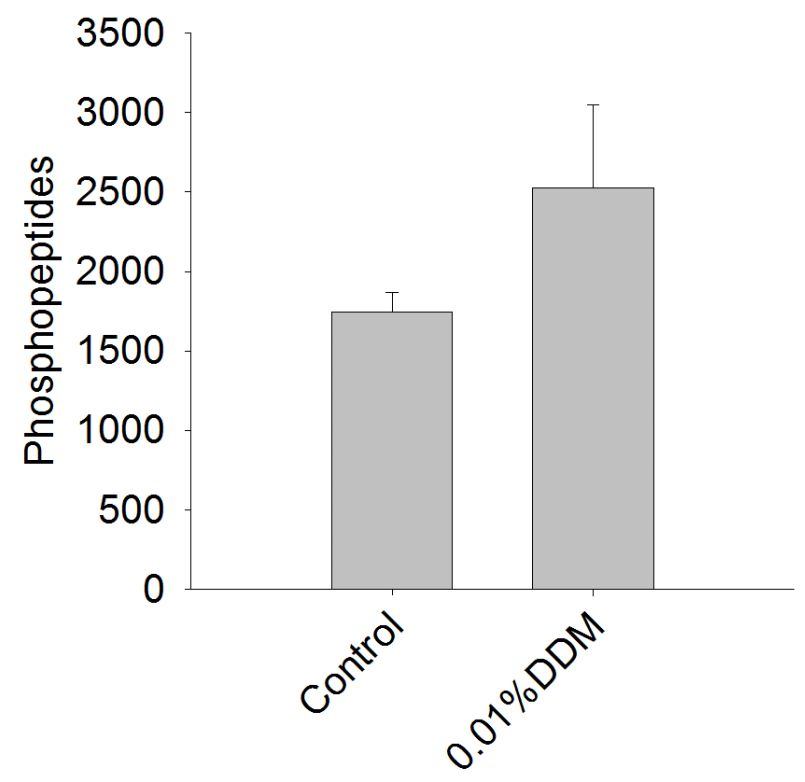

Fig. S4 The number of identified phosphopeptides eluted into the sample vial without and with $0.01 \%$ DDM as additive in collection buffer. The starting material was $20 \mu \mathrm{g}$ MCF-7 tryptic peptides. Error bars indicate mean $\pm \mathrm{SD}$. 